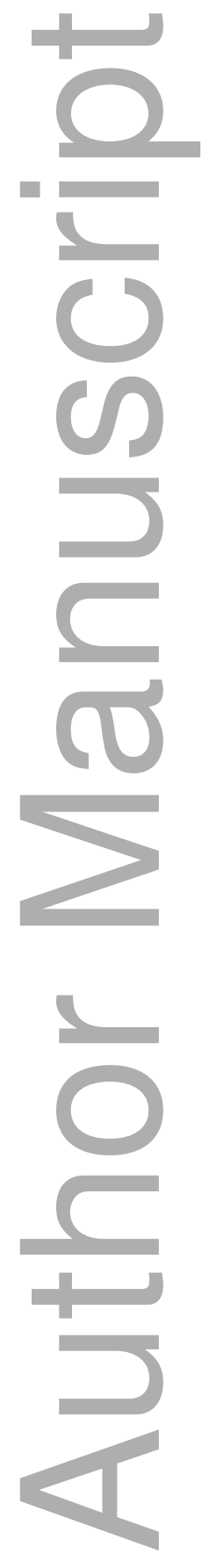

This is the author manuscript accepted for publication and has undergone full peer review but has not been through the copyediting, typesetting, pagination and proofreading process, which may lead to differences between this version and the Version of Record. Please cite this article as doi: 10.1111/anzs.12197

This article is protected by copyright. All rights reserved 


\section{The Size Accuracy of Combination Tests}

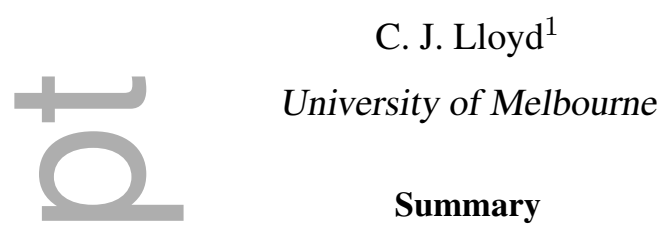

Adaptive clinical trials typically involve several independent stages. The $p$-values from each stage are synthesized through a so-called combination function which ensures that the overall test will be valid if the stagewise tests are valid. In practice however, approximate and possibly invalid stagewise tests are used. This paper studies how imperfections of the stagewise tests feed through into the combination test. Several general results are proven including some for discrete models. An approximation formula which directly links the combined size accuracy to the component size accuracy is given. In the wider context of adaptive clinical trials, the main conclusion is that the basic tests used should be size accurate at nominal sizes both much larger and also much smaller than nominal desired size. For binary outcomes, the implication is that the parametric bootstrap should be used.

Key words: adaptive designs; bootstrap $p$-value; Fisher combination; Laplace approximation; multi-stage design; normal combination; saddlepoint approximation;

\section{Introduction}

Consider a clinical trial designed to assess the effect of a treatment on some endpoint/response of interest. The treatment is considered effective if the mean response $\mu_{1}$ with the treatment exceeds the mean response $\mu_{0}$ with a possibly active control. There are several standard test statistics for measuring evidence for such a conclusion. For instance, when the endpoint is binary the means $\mu_{i}$ are just the probabilities of response and common test statistics are the pooled Z-test and the likelihood ratio (LR) test.

The test statistic is converted into a $p$-value, almost always on the basis of a distributional approximation, and the treatment is deemed effective at a significance level $\alpha$ if the $p$-value is less than or equal to $\alpha$, with the aim that the probability of falsely concluding that the treatment is effective (the type 1 error, also called test size) should equal $\alpha$. In fact, for standard methods this probability is sometimes not especially close to $\alpha$. It may also depend on parameters unspecified under the null.

Is size control really a practical problem? The left panel of Figure 1 displays typical size for some well-known standard tests of treatment effect at nominal significance level $\alpha=0.025$ for a binary response. The size depends on the unspecified baseline probability of response $p_{0}$. Henceforth, a plot of size versus any free nuisance parameter is called a size profile. Similar plots appear in Lloyd (2014) and the conclusion is that true size can far exceed nominal for the standard methods, even for quite large sample sizes. The right panel is for the so-called parametric bootstrap test which here, and in almost all cases, has type 1 error probability consistently close to nominal. Thus standard tests can perform very poorly with respect to size control but there is a general and computable alternative.

\footnotetext{
1200 Leicester Street, Carlton, 3053, AUSTRALIA. email: c.lloyd@mbs.edu
} 

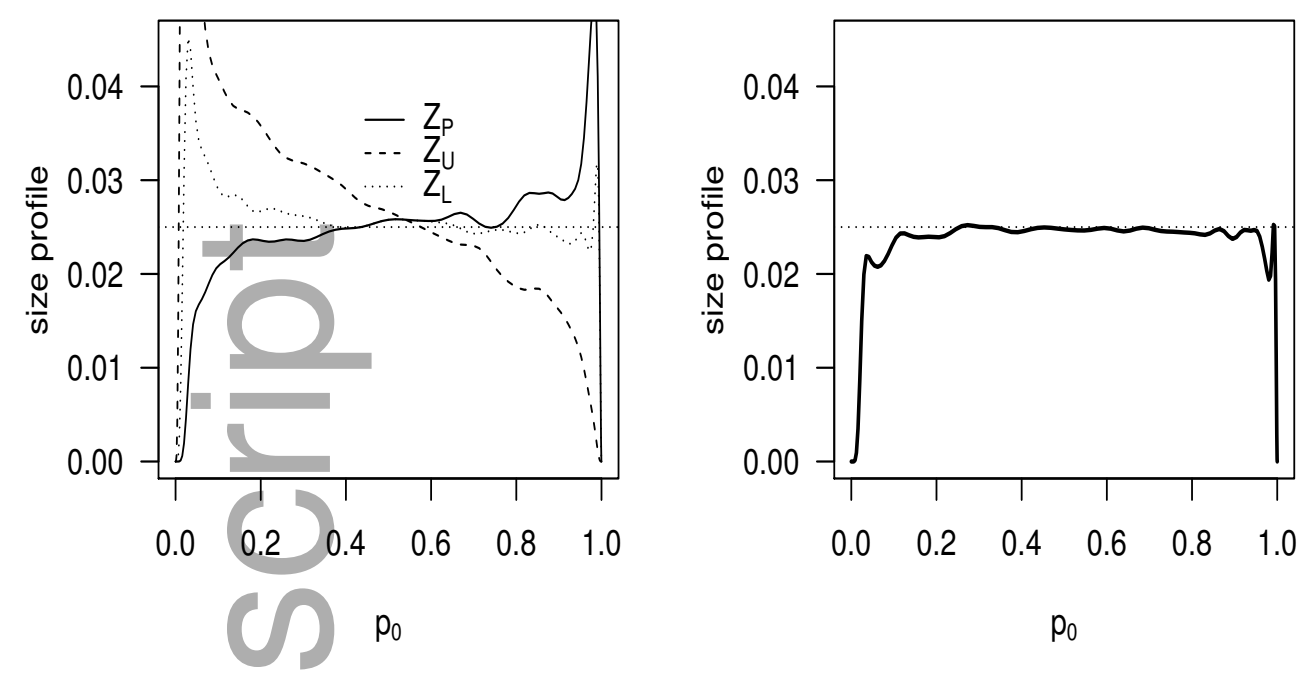

Figure 1. Size profiles of four tests of no-treatment effect. In both cases we are testing whether response under treatment $p_{1}$ exceeds response with control $p_{0}$ at nominal significance level 0.025 , from sample sizes $n_{0}=75$ for the control group and $n_{1}=175$ for the treatment group. The exact size $\alpha\left(p_{0}\right)$ is plotted against the free parameter $p_{0}$ under the null hypothesis that $p_{1}=p_{0}$. Left Pooled-z statistic, unpooled-z statistic and signed root of the LR statistic. Right. Parametric bootstrap test defined later in equation 3 .

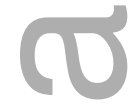

Within an adaptive design, there is often selection of the most promising treatment(s) at the first stage. Selection needs to be accounted for with some kind of multiple comparison adjustment (Simes, 1986) as well as the closed testing principle (CTP) if family-wise error is to be controlled (Marcus et al., 1976). These adjustments ensure that if the test employed for each treatment arm controls type 1 error then so does the adjusted test. The selected treatments are carried forward and tested again, perhaps several times with further selection and other adaptations, for instance with respect to sample size. Evidence across multiple stages is summarised through a so-called combination function (see Section 2). Again, this function is chosen so that provided each stage controls the error rate, so does the combined test. Yet we have just seen that standard single stage tests do not have exact size equal to $\alpha$.

Lloyd (2014) looked at how these violations of size control feed through multiple comparison adjustments with or without the CTP. Here we focus on combination across stages. The central issue we study is the extent to which combination tests suffer from the imperfections of the stage-wise tests. Does combination tend to mitigate or exaggerate any deficiencies? If the combination test controls size as poorly as the stage-wise test, then what is to be done?

The plan of the paper is as follows. The next section introduces the idea of a combination function. Section 3 establishes some unproven results on how the properties of the stagewise tests affect the size accuracy of combination tests under fairly restrictive conditions and illustrates them on some hypothetical parametric examples. In Section 4, we address the same issue under more general conditions by resorting to a Laplace approximation. This analysis shows that for combinations tests the component tests need to be size accurate at nominal sizes considerably larger than the ultimately desired size. In Section 5 we investigate 
application of the theory to a binary clinical trial and in Section 6 we apply the theory to a real example involving selection of the best of four treatments in a multi-stage adaptive design.

\section{Combination tests}

A function $C:[0,1]^{K} \rightarrow[0,1]$, increasing in each of the $K$ arguments, is a combination function if $C\left(P_{1}, \ldots, P_{K}\right)$ is uniformly distributed whenever $P_{1}, \ldots, P_{k}$ are independent and uniformly distributed. The practical purpose of combination functions is to combine the $p$ values $P_{j}$ calculated on $K$ independent experiments into a single $p$-value.

The most common combination is the normal combination (Mosteller, 1954). If each $p$-value $P_{j}$ is uniform then $Z_{j}=\Phi^{-1}\left(1-P_{j}\right)$ is standard normal and so the combined test statistic $Z_{N}=\sum Z_{j} / \sqrt{K}$ is standard normal. When the information content differs across experiments, a weighted version of the combination test can be used. The main competing method (Fisher, 1932) is based on the idea that if each $p$-value $P_{j}$ is uniform then $T_{j}=-\log P_{j}$ is exponential so that $\sum T_{j}$ is gamma distributed with shape parameter $K$. The combined $p$-value is the probability that $\sum T_{j}$ exceeds its observed value. Neither the Fisher nor normal combination dominates the other with respect to power; the power depends on how the non-null distribution of the component $p$-values deviates from uniform. The normal combination tends to be smaller when all $K p$-values are small while the Fisher combination, unlike the normal, will definitely reject if just one of the $p$-values is small enough. Both lose a small amount of power (Bank et al, 1996; Lin \& Zeng, 2010) compared to full analysis of the joint likelihood.

The context of this study of combinations is as an ingredient in the analysis of socalled adaptive designs, where sample size or even hypotheses can be adapted on the basis of interim results; see Jennison \& Turnbull (2007) for a review. If there is treatment selection then some kind of multiple comparison adjustment is required, but this is not the topic of this paper. The analysis of such designs typically employs the Fisher or normal combination at the appropriate point. Some authors such as Bauer \& Kohne (1994) have favoured the Fisher combination while others such as Lehmacher \& Wassmer (1999) prefer the normal combination. We are not primarily interested in which combination should be used here. Rather, the focus is on how inaccuracies of the basic tests feed through to inaccuracies of the combined test, with emphasis on the normal combination, because of its relative tractability. The next section gives some general results on size bias of combinations.

\section{Size accuracy of combination test families}

A test is called conservative (liberal) at nominal size $\alpha$ if the true size is smaller (larger) than $\alpha$ and exact if it is exactly equal to $\alpha$. The defining property of a combination function is that it converts independent exact tests into an exact test. It seems obvious then that conservative/liberal tests would remain so after combination. However, the issue is not so simple as first appears.

Firstly, discreteness complicates the definition of stochastic dominance which is the usual basis for defining a test as being exact/conservative/liberal. Secondly, for adaptive multi-stage trials the stage-wise $p$-values are typically not independent, whereas the defining property of a combination function is expressed in terms of independent $p$-values. We allow for both of these complications in this section. 
The more challenging issue is that tests are sometimes neither liberal nor conservative, namely when they are liberal at some nominal sizes and conservative at others. This situation requires approximate methods that essentially average over different nominal sizes and is discussed in Section 4. For now, we look at the simpler case where the test is either conservative/exact/liberal for all levels $\alpha$, in which case we call it a conservative/exact/liberal test family. For continuous tests, this is equivalent to the $p$-value statistic being stochastically larger than/equal to/smaller than uniform.

Theorem 1 (continuous combination). Let $P_{1}, P_{2}, \ldots, P_{k}$ be a sequence of continuous variables on $[0,1]$. If, conditional on $P_{1}, \ldots, P_{j-1}$, each $P_{j}$ defines a conservative/exact/liberal family then the combination test is a conservative/exact/liberal family.

Theorem 2 (discrete combination). Let $P_{1}, P_{2}, \ldots, P_{k}$ be a sequence of discrete variables on [0,1]. If, conditional on $P_{1}, \ldots, P_{j-1}$,

(A) each $P_{j}$ is exact then the combination test is a conservative family.

(B) each $P_{j}$ is defines a conservative family then the combination test is a conservative family.

All details and proofs of the above assertions are given in Web Appendix A. In some sense, the results are obvious, certainly for the continuous case. However for discrete tests the "obvious" result that liberal test families are liberal after combination is untrue; see the example following the proof of Theorem $2 \mathrm{~A}$ in the Web Appendix. In practice, as the support of the $p$-values becomes finer, these discreteness effects become smaller and we expect to see the clean results for continuous tests hold to a high level of approximation.

These results do not say how conservative or liberal a non-exact combination test might be. We conclude this section by looking at three parametric models for the distribution of a $p$-value that generate liberal or conservative test families. All three models have the property that the size bias is amplified by combination. These same models will be used to illustrate our approximation formula in Section 4.

Example 1. Power distribution. Suppose that the $p$-value $P=\Phi(-Z)$ has the distribution function $F_{P}(p)=p^{1+\theta}$ which is stochastically larger/smaller than uniform if $\theta$ is larger/smaller than 0 . The single stage test of target size $\alpha$ then has actual size $\alpha^{1+\theta}$ which defines a conservative/liberal family as $\theta$ is smaller/larger than 0 . Thus, by Theorem 1 , the combination test will be conservative/liberal. Using a general expression in Web Appendix $\mathrm{B}$, the size of the $K=2$ stage normal combination test of nominal size $\alpha$ is given exactly by

$$
\tau_{2}(\alpha)=\int_{0}^{1} \Phi\left(\Phi^{-1}\left(1-(1-y)^{1 /(1+\theta)}\right)-q_{\alpha} \sqrt{2}\right) d y
$$

This is easy to compute numerically and is plotted in the left panel of Figure 2.

Example 2. Non-unit scale exponential. Recalling the motivation for Fisher's combination, suppose that each $T_{j}=-\log P_{j}$ is exponential with scale $1+\delta$ rather than 1 . Under this assumption, the sum has the gamma distribution with scale $1+\delta$. It is not difficult then to show that the size of the Fisher combination test is

$$
1-\Gamma\left(K,(1+\delta) \Gamma^{-1}(K, 1-\alpha)\right)
$$




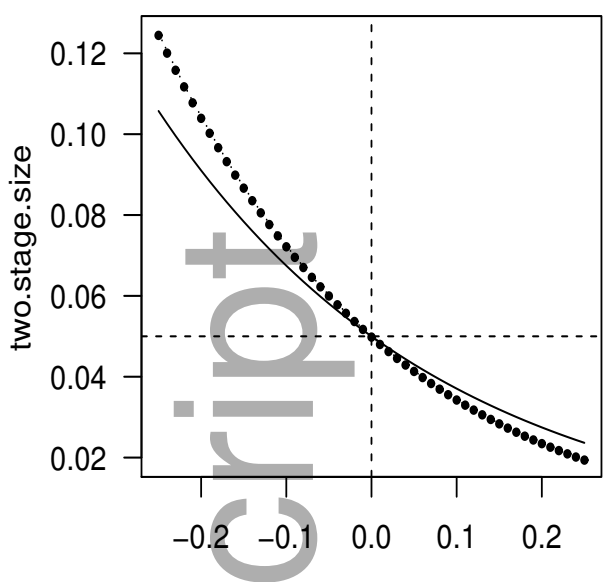

$\theta$

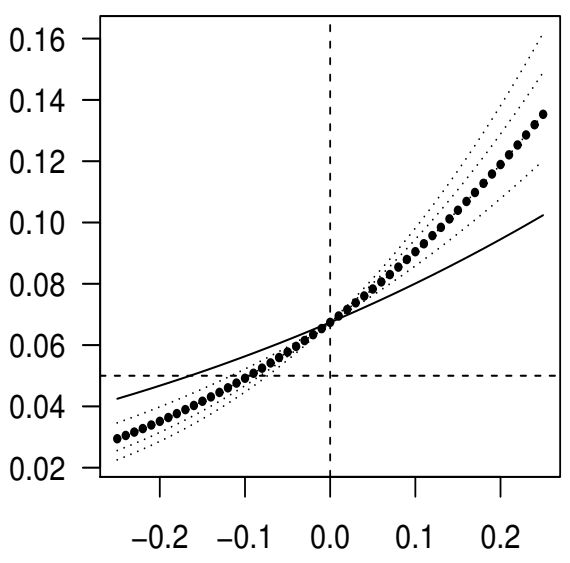

$\mu$

Figure 2. Size $\tau_{K}(\alpha)$ of $K$-combination tests. The size of a single stage test is a solid line and those of the multi-stage tests are dotted lines. The Laplace approximation given in equation 2 is displayed as filled points. Left. Power model. The exact size of the nominal $\alpha=0.05$ test of $\theta>0$ for the power model of Example 1. The Laplace approximation to the 2-stage combination is indistinguishable from the true size. Right. Normal shift model. The exact size $\tau_{K}(0.05)$ of the nominal $\alpha=0.05$ test of the mean $\mu>0$ as a function of mean $\mu$ for $K=1, \ldots, 5$ when $\sigma=1.1$. The Laplace approximation to the 3 -stage combination is again indistinguishable from the true size.

which, by well known monotonicity properties of the incomplete gamma function, is monotone increasing/decreasing in $K$ when $\delta$ is greater/smaller than 0 .

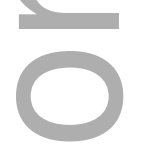

Example 3. Normal shift. Suppose that the Z-statistics have the normal distribution but with mean $\mu$ not necessarily equal to zero. If $\mu$ is positive/negative then this defines a conservative/liberal family. Thus, the combination test will be conservative/liberal and it is easy to show that the true size $\tau_{K}(\alpha)$ of the nominal of size $\alpha$ normal combination test is $\tau_{K}(\alpha)=\Phi\left(\mu \sqrt{K}+\Phi^{-1}(\alpha)\right)$. Clearly bias increases as $K$ increases unless $\mu=0$.

Consider now when $\sigma=1.1$ rather than the nominal value 1.0. For fixed values of $\mu$, this is no longer a conservative or liberal family; for instance, for small values of $\alpha$ it tends to be liberal even if $\mu<0$. Therefore the previous theory does not apply and we see that a combination of liberal tests need not be liberal. It can easily be shown that $\tau_{K}(\alpha)=\Phi\left(\left(\mu \sqrt{K}+\Phi^{-1}(\alpha)\right) / \sigma\right)$. This is plotted in the right panel of Figure 2 for $K=1$ to 5 . Broadly speaking, bias increases with $K$. However, when $\mu=-0.1$ the single stage test is liberal but becomes less so with combination and has true size 0.0492 which is almost exactly nominal when $K=4$. For larger values of $K$ it is conservative. 


\section{Approximation to the size of a normal combination test}

It is not uncommon that tests will be conservative at some nominal sizes and liberal at others. The question arises then of whether the combination test will follow the same pattern. We now study this issue, for the normal combination only.

As before, let $Z_{i}=\Phi^{-1}\left(1-P_{i}\right)$ be the stage-wise test statistics with nominal standard normal distribution. The actual distribution and density functions are denoted by $H_{1}$ and $h_{1}$ respectively. Throughout we denote the survivor function by $S_{1}(z)=1-H_{1}\left(z^{-}\right)$. Thus the true size of the one stage test is $\tau_{1}(\alpha)=S_{1}\left(q_{\alpha}\right)$ where $q_{\alpha}=-\Phi^{-1}(\alpha)$. Alternatively we can re-express this as $S_{1}(q)=\tau_{1}(\Phi(-q))$.

Let $S_{2: K}, H_{2: K}, h_{2: K}$ denote the survivor, distribution and density functions of the standardised sum $\left(Z_{2}+\ldots+Z_{K}\right) / \sqrt{K-1}$. The normal combination rejects the null hypothesis exactly when

$$
\left(Z_{1}+\ldots+Z_{K}\right) / \sqrt{K}>q_{\alpha} \Leftrightarrow Z_{1}>\sqrt{K} q_{\alpha}-\sqrt{K-1}\left(\frac{Z_{2}+\ldots+Z_{K}}{\sqrt{K-1}}\right) .
$$

Hence the probability that the combined test rejects the null hypothesis is

$$
\tau_{K}(\alpha)=S_{K}\left(q_{\alpha}\right)=\int_{-\infty}^{\infty} S_{1}\left(q_{\alpha} \sqrt{K}-z \sqrt{K-1}\right) h_{2: K}(z) d z
$$

which is the mean of the size of the single stage test of nominal size $\Phi\left(z \sqrt{K-1}-q_{\alpha} \sqrt{K}\right)$ where $z$ is weighted according to $h_{2: K}$. Not only are some values of $z$ weighted more heavily than others but it turns out that the expression depends largely on a single value of $z$, namely the turning point in a Laplace approximation.

The integrand in (1) can be expressed as $\exp \left\{-g_{\alpha}(z)\right\}$ where

$$
g_{\alpha}(z)=-\log \left\{S_{1}\left(q_{\alpha} \sqrt{K}-z \sqrt{K-1}\right)\right\}-\log h_{2: K}(z) .
$$

Let $\hat{z}_{\alpha, K}$ be the solution for $z$ of

$$
g_{\alpha}^{\prime}(z)=-\frac{h_{2: K}^{\prime}(z)}{h_{2: K}(z)}-\sqrt{K-1} \frac{h_{1}\left(q_{\alpha} \sqrt{K}-z \sqrt{K-1}\right)}{S_{1}\left(q_{\alpha} \sqrt{K}-z \sqrt{K-1}\right)}=0 .
$$

Next define the quantile $\hat{Q}_{\alpha, K}=q_{\alpha} \sqrt{K}-\hat{z}_{\alpha, K} \sqrt{K-1}$ and $\hat{A}_{\alpha, K}=\Phi\left(-\hat{Q}_{\alpha, K}\right)$ the nominal size associated with that quantile. With this condensed notation, the Laplace approximation to (1) is given by Butler (2007, p.42) as

$$
\tau_{K}(\alpha) \approx \frac{\sqrt{2 \pi} e^{-g_{\alpha}\left(\hat{z}_{\alpha, K}\right)}}{\sqrt{g_{\alpha}^{\prime \prime}\left(\hat{z}_{\alpha, K}\right)}}=\frac{\sqrt{2 \pi} h_{2: K}(\hat{z})}{\sqrt{g_{\alpha}^{\prime \prime}(\hat{z})}} \tau_{1}(\hat{A})
$$

where we have dropped the subscripts $(\alpha, K)$ in the last expression. An analogous expression can be given for two-tailed tests by approximating the upper and lower tails separately, see Web Appendix D for some details.

This equation reveals the main connection between the size bias of the single stage and multi-stage test. To the extent that the approximation is accurate (which will be evaluated in coming examples), the size of the nominal size $\alpha$ combination test mainly depends on the 
size of the one stage test not at nominal size $\alpha$ but at nominal size $\hat{A}_{\alpha, K}$. All other things being equal, if the single stage test of nominal size $\hat{A}_{\alpha, K}$ has size $20 \%$ higher than nominal, we expect that the multi-stage test will have size $20 \%$ higher than $\alpha$. While the leading term is not invariant to deviations of the distribution of the $Z_{i}$ from nominal standard normal, there is no implication that such deviations would make this leading term larger or smaller.

Example 1 (continued). With the one stage distribution $H_{1}(z)=1-(1-\Phi(z))^{1+\theta}$, the Laplace approximation (2) to the true size of the combination test with $K=2$ is

$$
\frac{(1+\theta) e^{-\hat{z}^{2} / 2}(1-\Phi(\hat{z}))^{\theta}}{\sqrt{g_{\alpha}^{\prime \prime}(\hat{z})}}\left(1-\Phi\left(q_{\alpha} \sqrt{2}-\hat{z}\right)\right)^{1+\theta}
$$

where $\hat{z}_{\theta, \alpha}$ satisfies the equation

$$
g_{\alpha}^{\prime}(z)=z+\theta \frac{\phi(z)}{1-\Phi(z)}-(1+\theta)\left\{\frac{\phi\left(q_{\alpha} \sqrt{2}-z\right)}{1-\Phi\left(q_{\alpha} \sqrt{2}-z\right)}\right\}=0 .
$$

The second derivative is complicated but follows directly from the above or (more easily) from a more general expression given in Web Appendix C with $\ell_{1}^{\prime}(z)=-z-\theta \phi(z) / \Phi(-z)$. This has been computed in the left panel of Figure 2 and is almost visually indistinguishable from the true function.

Example 3 (continued). Let $\phi_{\sigma}, \Phi_{\sigma}$ denote the density and distribution of a normal random variable with mean zero and standard deviation $\sigma$. When $Z$ is normal with mean $\mu$ and standard deviation $\sigma$, we have $H_{K}(z)=\Phi_{\sigma}(z-\mu \sqrt{K})$ and the function $g_{\alpha}(z)$ is given by

$$
g_{\alpha}(z)=-\log \Phi_{\sigma}\left(z \sqrt{K-1}+\mu-q_{\alpha} \sqrt{K}\right)-\log \phi_{\sigma}(z-\mu \sqrt{K-1})
$$

whence

$$
g_{\alpha}^{\prime}(z)=\frac{z-\mu \sqrt{K-1}}{\sigma}-\sqrt{K-1} \frac{\phi_{\sigma}\left(z \sqrt{K-1}+\mu-q_{\alpha} \sqrt{K}\right)}{\Phi_{\sigma}\left(z \sqrt{K-1}+\mu-q_{\alpha} \sqrt{K}\right)} .
$$

The Laplace approximation to the size of the combination test is then

$$
\frac{\sqrt{2 \pi} \phi(\hat{z}-\mu \sqrt{K-1})}{\sigma \sqrt{g_{\alpha}^{\prime \prime}(\hat{z})}} \Phi_{\sigma}\left(\hat{z} \sqrt{K-1}+\mu-q_{\alpha} \sqrt{K}\right),
$$

where $\hat{z}(\alpha, \mu, K)$ satisfies $g_{\alpha}^{\prime}(z)=0$. This has been computed and plotted for $K=3$ in the right panel of Figure 2 and is again almost visually indistinguishable from the true function. It is of course well known that Laplace approximations are usually highly accurate.

Example 4. Normal mixture. In the previous example, the single stage test was neither a conservative nor a liberal family so conservative tests may become liberal when combined. The current example illustrates this fact in more detail and relates the liberality of the two stage to the liberality of the one stage test at the Laplace relevant size. Suppose that the Z statistic for each stage does not follow its nominal standard normal distribution but is instead a normal mixture of the form

$$
h_{1}(z)=(1-p) \phi(z)+p \phi\left(\left(z-q^{*}\right) / \sigma\right) / \sigma .
$$


The left panel of Figure 3 shows this density function when $b=0.1, q^{*}=0.8, \sigma=0.4$ superimposed on the nominal standard normal density function. The true size of the nominal size $\alpha$ test is

$$
\tau_{1}(\alpha)=(1-p) \alpha+p \Phi\left(\frac{\left.q^{*}+\Phi^{-1}(\alpha)\right)}{\sigma}\right) .
$$

For nominal size $\alpha=0.05$, the test is conservative with true size $\tau_{1}(.05)=0.0479$ so that there is a relative bias of 0.959 . For other nominal sizes the test can be liberal. The right panel of Figure 3 displays a plot of $\tau_{1}(\alpha) / \alpha$ against $\alpha$. The solid vertical line is at $\alpha=0.05$. The one-stage test is conservative for nominal sizes less than 0.0692 and liberal otherwise.
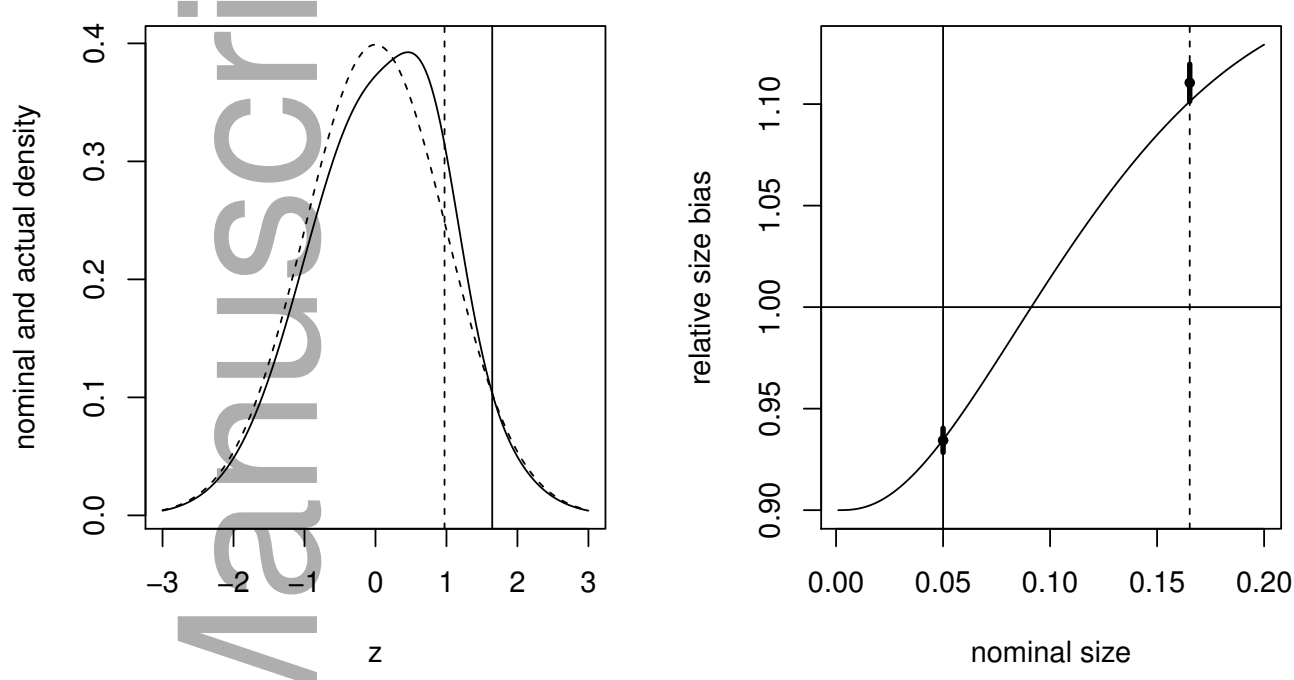

Figure 3. Contaminated mixture model. Left. Nominal standard normal and true contaminated density. Laplace relevant quantile $\hat{Q}_{0.05,2}=0.973$ is displayed as a dotted vertical line. Right. Relative size bias versus nominal size of a single stage test with simulations results of a two stage test of nominal size $\hat{A}_{0.05,2}=0.165$

To determine $\hat{z}$ we find the root of

$$
g_{\alpha}^{\prime}(z)=\frac{(1-p) z \phi(z)+\frac{p}{\sigma} \frac{z-q^{*}}{\sigma^{2}} \phi\left(\frac{z-q^{*}}{\sigma}\right)}{(1-p) \phi(z)+\frac{p}{\sigma} \phi\left(\frac{z-q^{*}}{\sigma}\right)}-\frac{(1-p) \phi\left(z-q_{\alpha} \sqrt{2}\right)+\frac{p}{\sigma} \phi\left(\frac{q^{*}+z-q_{\alpha} \sqrt{2}}{\sigma}\right)}{(1-p) \Phi\left(z-q_{\alpha} \sqrt{2}\right)+p \Phi\left(\frac{q^{*}+z-q_{\alpha} \sqrt{2}}{\sigma}\right)} .
$$

For $\alpha=0.05$ the root is $\hat{z}_{0.05,2}=1.353$ so $\hat{Q}_{0.05,2}=0.973$ and $\hat{A}_{0.05,2}=0.165$. The dotted vertical line in the right panel of Figure 3 is at this Laplace relevant size of 0.165 . According to the previous theory, we predict that the two-stage combination will be liberal with a relative size bias of $\tau_{1}(0.165)=1.101$, even though the one-stage test is conservative. A simulation (95\% confidence interval indicated on plot) reveals that the actual size of the two-stage combination is 0.0555 which is a relative bias of 1.110 .

\section{Binomial trials}

Suppose that $n_{0}$ subjects are randomly assigned to the control group of whom $y_{0}$ respond and the remaining $n_{1}$ subjects are assigned to the treatment group of whom $y_{1}$ respond. The 
theoretical probabilities of response are $p_{0}$ for the control group and $p_{1}$ for the treatment group and we want to test the alternative hypothesis $p_{1}>p_{0}$ against the null hypothesis $p_{1} \leq p_{0}$. There are many test statistics that have been proposed in the literature and it is also well known that the size control of standard first order methods can be extremely poor. It is not our intention to describe the dozens of statistics that have been suggested. Rather, our purpose is to see how the previous theory may apply to one example.

\subsection{Discrete models}

For discrete models the integral expression (1) can be interpreted as a sum, and under this interpretation still provides an exact expression for $\tau_{K}(\alpha)$. The question is how to apply the Laplace approximation in this context since there is no continuous function $g(x)$.

Laplace approximations are often applied to means of functions of variables distributed on a lattice and can be motivated by extending the discrete variable into a continuous one and arguing that the resulting integral will still be close to the sum. This often involves a continuity correction, see for instance section 1.2.3 and 1.2.4 of Butler (2007). In short, application of Laplace approximations to discrete distributions on a lattice is common and successful.

We are not able to prove that (2) can be applied to the standard test statistics that are use for testing treatment effects from binomial trials. For general discrete variables the sum can still be approximated by a continuous integral, but details of the continuity correction are hard to specify or implement. First, it would be necessary to determine the support of the distribution in question which can be difficult. Second, for many practical situations the support of the variable is dense enough that the correction would have little effect. A case in point is the combination of test statistics for binary endpoint trials with $\left(n_{0}, n_{1}\right)$ subjects. The number of points in the support is of order $n_{0} n_{1}$ which is $O\left(n^{2}\right)$ if both $n_{0}$ and $n_{1}$ are $O(n)$. Within a range of typical statistical deviation the number of points in the support will be $O\left(n^{3 / 2}\right)$. For combinations of $K>2$ statistics, the support is even finer.

\subsection{Standard tests}

There are three standard test statistics in common use. The unpooled/pooled Z statistics $Z_{U} / Z_{P}$ are based on the maximum likelihood (ML) estimate and its variance, estimated freely or under the null respectively. The other common statistic is the signed version $Z_{L}$ of the LR statistic. Explicit formulas are available in any textbook. Each of the statistics $Z_{U}, Z_{P}, Z_{L}$ has asymptotic standard normal distribution function $\Phi$ for large sample sizes provided neither $p_{0}$ nor $p_{1}$ equal 0 or 1 . For each, large values support the alternative hypothesis that $p_{1}>p_{0}$ and they are converted into an (approximate) $p$-value via the formula $P=1-\Phi(Z)$. Equivalently, the null hypothesis is rejected if $Z$ exceeds the upper quantile $q_{\alpha}$ of the normal distribution.

How accurate are these test statistics under typical conditions? The left panel of Figure 1 displays the size profiles of these three statistics for $n_{0}=75, n_{1}=175$ where the nominal size is $\alpha=0.025$. Even for these quite large sample sizes, the true size can exceed the nominal by a large factor. Much but not all of the violation occurs for extreme baseline rates. The pooled statistic seems most satisfactory of the three, though this is not always the case. Another oft cited example with samples sizes 283 and 47 is given in Berger \& Boos (1994). A large scale numerical study in Lloyd (2013) demonstrated that even for quite large sample sizes, standard statistics typically control size poorly. 


\subsection{Parametric bootstrap test}

A more accurate, albeit more complex, statistic is obtained by choosing a basic statistic $Z$ and then calculating the exact significance of its observed value $z_{\mathrm{obs}}$ of $Z$. This is given by

$$
P_{B}=\sum_{y_{0}=0}^{n_{0}} \sum_{y_{1}=0}^{n_{1}} \mathbf{1}_{\left\{Z\left(y_{0}, y_{1}\right) \geq z_{\mathrm{obs}}\right\}} B\left(y_{0} ; n_{0}, \hat{p}_{0}\right) B\left(y_{1} ; n_{1}, \hat{p}_{1}\right)
$$

where $\mathbf{1}$ is the indicator function, $B(y ; n, p)$ is the probability function of the $\operatorname{binomial}(n, p)$ distribution and $\left(\hat{p}_{0}, \hat{p}_{1}\right)$ are the ML estimates of $\left(p_{0}, p_{1}\right)$ under the null hypothesis. When the null is $p_{0} \leq p_{1}$, these both equal the overall event rate. I call this the parametric bootstrap $p$-value although for reasonable sample sizes no simulation is required to calculate it. It is worth noting that even though $P_{B}$ is computed after an initial choice of the test statistic $Z$, this choice has very little effect on the final computed $p$-value (Lloyd, 2011) which is another point in favour of $P_{B}$.

The size profile of the bootstrap $p$-value is shown in the right hand panel of Figure 1 and is very close to nominal for almost all values of $p_{0}$. Such favourable properties are not specific to the choice of $n_{0}, n_{1}$ and $\alpha$ (Lloyd, 2013) and so there is no need in practice to compute the size profile; it can be assumed to be favourable. The bootstrap $p$-value for the observed data set can be computed in a fraction of a second for samples sizes up to 1000 .

\subsection{Size bias of 2-stage combination}

Figure 4 shows typical results for the case $n_{0}=30, n_{1}=40$ and $\alpha=0.05$. Sample sizes are assumed to be fixed and the same for each stage. Each plot gives the size profile divided by the nominal size, so that a reference value of 1 means the test is neither conservative nor liberal. The three profiles correspond to the normal and Fisher combinations of size 0.05 and the single stage test of Laplace relevant size $\hat{A}_{0.05,2}=0.187$. The left plots are for the LR statistic and the right plots are for the bootstrap test. The Fisher and normal profiles seem very similar so, even though our theory applies only to the combination, it is also predictive of the Fisher size profile in this example. The size bias is again amplified by combination in this instance. The combined bootstrap tests share the nice properties of the single stage bootstrap tests and there is almost no size bias, except near the extremes of the parameter space where the test is conservative. This is almost always the case for bootstrap tests for binomial trials; see Lloyd (2013).

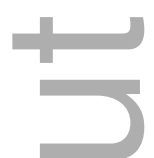

\section{Example}

The following data are from a real adaptive clinical trial recently conducted in Europe and North America. Confidentiality restrictions prevent disclosure of details of the disease or treatment but the binary response here was a blood-based measure of improvement. The null hypothesis is that the treatment has no effect on response and there were four variations of the treatment considered at the first stage. The variant with the smallest $p$-value would be carried through to the second stage.

This design is fairly standard and a correct $p$-value must combine the four treatment $p$ values at the first stage with the $p$-value from the selected treatment at the second stage, taking 

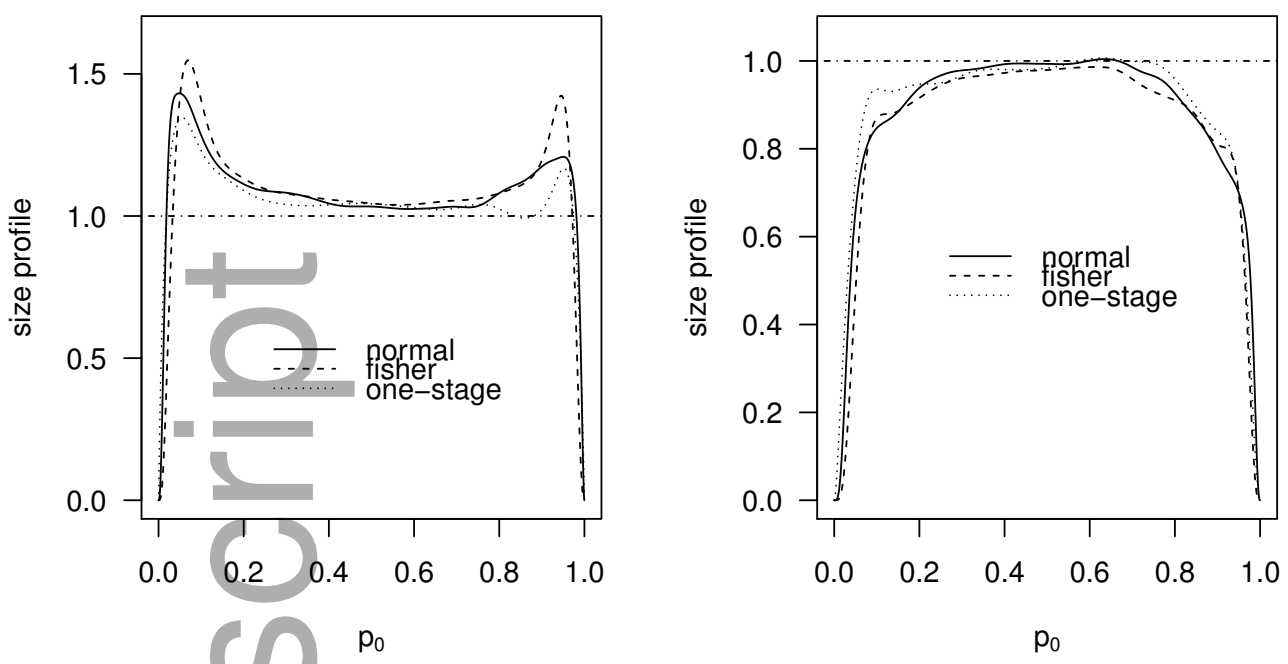

Figure 4. Relative size profiles of LR based tests. Exact size of one and two stage combination tests of $p_{1}=p_{0}$ versus $p_{1}>p_{0}$ for sample sizes $n_{0}=30, n_{1}=40$ and $\alpha=0.05$. Each profile has been divided by nominal size so a value of 1.0 means exactly size unbiased. For the normal and Fisher combinations nominal size is $\alpha=0.05$. For the one-stage test the nominal size is the Laplace relevant size 0.187 corresponding to $\alpha=0.05$. Left. LR tests Right. Bootstrap tests.

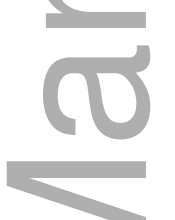

account of both selection and combination across stages. While this paper is concerned only with combination, Lloyd (2014) looked at selection and it seems pertinent to place the current work within the most likely context where it would be employed.

For each of the five comparisons, we have calculated $p$-values based on the pooled and unpooled $Z$ tests and on the LR as well as on the bootstrap method applied to the LR test in Table 1. At the first stage, the smallest $p$-value was 0.028 for treatment $\mathrm{D}$. This can be adjusted to the Bonferroni $p$-value $4 \times 0.028=0.113$. A more powerful adjustment is the Simes (1986) $p$-value which equals the Bonferroni $p$-value in this instance, but can potentially be smaller. We then combine the first stage $p$-value 0.113 with the second stage $p$-value 0.033 using the normal combination which gives 0.016. Using the Fisher combination the result is 0.025 . There is a large literature on the power characteristics of different combinations. Suffice it to say that neither Fisher nor normal has a uniform power advantage over the other. Of course, the researcher may not choose the smaller of the two after seeing the result without seriously violating the size restriction.

These calculations are repeated for the unpooled z-test, the LR test and the bootstrap test. Only the latter is reliable in terms of size control. In this example, bootstrap $p$-values and pooled-z $p$-values happen to be close but this is certainly not always the case. Nor are bootstrap $p$-value always larger than all the approximate $p$-values though often they are. According to Figure 4, for samples of size of the order of 30 and 40 (which is similar to the sample sizes in the example) the LR statistic and unpooled z-test can be severely liberal. The more unbalanced the sample, the less reliable is the pooled test. 
Table 1. Results of a four arm adaptive trial. The upper section is for the first stage where four treatments were compared with a control. Stage 1 Overall the $p$-value is based on the Simes multiple comparison of the four treatment $p$-values, which in each case is four times the smallest (though this is not always the case). The lower section is for the second stage trial where the selected treatment $\mathrm{D}$ is compared with the control. The last two lines combine the stage 1 and stage $2 p$-values using normal and Fisher combination functions. The $p$-values in the columns 4-6 are based on the standard approximate tests described in the text, and the bootstrap $p$-values were given explicitly in equation 3 .

\begin{tabular}{lcc|cccc} 
& & & & & & \\
Treatment & Response & Patients & Unpooled & Pooled & LR & Bootstrap \\
\hline Stage 1 Control & 7 & 30 & & & & \\
Treatment A & 11 & 41 & 0.368 & 0.369 & 0.276 & 0.383 \\
Treatment B & 11 & 40 & 0.345 & 0.347 & 0.257 & 0.360 \\
Treatment C & 13 & 39 & 0.177 & 0.182 & 0.123 & 0.191 \\
Treatment D & 19 & 42 & 0.022 & 0.028 & 0.015 & 0.030 \\
Stage 1 Overall & & & 0.088 & 0.113 & 0.060 & 0.120 \\
\hline Stage 2 Control & 10 & 40 & & & & \\
Stage 2 Treatment (D) & 25 & 58 & 0.028 & 0.033 & 0.031 & 0.034 \\
\hline Combined (Normal) & & & 0.010 & 0.016 & 0.008 & 0.017 \\
Combined (Fisher) & & & 0.017 & 0.025 & 0.014 & 0.027
\end{tabular}

\section{Discussion}

Inference from many clinical trial designs requires adjustment of $p$-values both for selection and combination. Virtually all the literature on inference for these designs assumes that the basic test statistics are exact. For instance, Magirr, Stallard \& Jaki (2014) assume that the data are exactly normally distributed with equal variance. A recent paper of Levin et al. (2014) makes the same assumption in evaluating the size and power of various adaptive sample size (i.e. sequential) designs. Many endpoints are not normally distributed, binary endpoints being the most extreme example.

Rather unsurprisingly, it turns out (Lloyd, 2014) that the properties of selection adjusted $p$-values for a level $\alpha$ test are largely determined by the properties of the basic $p$-values at level $\alpha / m$, where $m$ is the number of treatment arms. The important conclusion is that the final test properties depends on the behaviour of the basic test at sizes much smaller than nominal. The focus of this paper is on combination of $p$-values across stages. The theory presented suggests that the size accuracy of the combined test will depend mainly on the size accuracy of the stage-wise tests, but at a larger nominal size than $\alpha$. Together, these results suggest that for adaptive tests to be size accurate, inference must be based on $p$-values that have very good size control at sizes both smaller and larger than the nominal target.

There are other highly accurate $p$-values that could be used instead of the bootstrap; for instance a guaranteed but conservative method could be applied, perhaps only when expected counts fall below some threshold. If computation costs are not an issue then so-called exact $p$-values based on maximisation with respect to the nuisance parameter may be used. An alternative is partial maximisation with respect to the nuisance parameter, as recommended by Berger \& Boos (1994). The key point is that feeding standard tests into combination functions may lead to poor size accuracy and makes a mockery of theory on which the combinations are based.

Supplementary material. Supplementary appendices (available from the online repository at the Wiley web site) include proofs of all assertions in Section 3 and some details of the Laplace approximation referred to in Section 4. 


\section{References.}

Bank, N., Kohne, P. \& BAuer, P. (1996). On the power of Fisher's combination test for two stage sampling in the presence of nuisance parameters. Biometrical Journal 38, 25-37.

BAUER, P. \& KoHne, P. (1994). Evaluation of experiments with adaptive interim analyses. Biometrics 50, 1029-1041.

Berger, R. L \& Boos, D. D. (1994). P-values maximized over a confidence set for the nuisance parameter. Journal of the American Statistical Association 89, 1012-1016.

Brazzale, A.R. Dayison, A.C., \& ReID, N. (2002). Applied asymptotics: case studies in small sample statistics. Cambridge: Cambridge University Press.

BUtLer, R. W. (2007). Saddlepoint approximations with applications. Cambridge: Cambridge University Press.

FISHER, R.A. (1932). Statistical methods for research workers. 4th ed. London: Oliver and Boyd.

Jennison, C \& Turnbull, B.W. (2007). Adaptive seamless designs: Selection and prospective testing of hypotheses. Journal of Biopharmaceutical Statistics 17, 1153-1161.

LEHMACHER, W. \& WASSMER, G. (1999). Adaptive sample size calculation in group sequential trials. Biometrics 55, 1286-1290.

LEVIN G.P., EMERSON S.C. \& EMERSON S.S. (2014). An evaluation of inferential procedures for adaptive clinical trial designs with pre-specified rules for modifying the sample size. Biometrics 70, 556-567.

LIN, D.Y. \& ZENG, D. (2010). On the relative efficiency of using summary statistics versus individual-level data in meta-analysis. Biometrika 97, 321-332.

LLOYD, C.J. (2011). How close are alternative bootstrap p-values? Statistics and Probability Letters 80, 1972-1976.

LLOYD, C.J. (2013). A numerical investigation of the accuracy of parametric bootstrap for discrete data. Computational Statistics and Data Analysis 61, 1-6.

LLOYD, C.J. (2014). On the exact size of tests of treatment effects in multi-arm clinical trials. Australian and New Zealand Journal of Statistics 56, 1-6.

MagirR A., Stallard N. \& JAKI, T. (2014). Flexible sequential designs for multi-arm clinical trials. Statistics in Medicine 33, 3269-3279.

Mosteller, F. \& Bush, R.R. (1954). Selected quantitative techniques. Handbook of Social Psychology. Cambridge MA: Addison Wesley.

MARCus, R., PERITZ, E. \& GABriel, K.R. (1976). On closed testing procedures with special reference to ordered analysis of variance. Biometrika 63, 655-660.

Rohmel, J. (2005). Problems with existing procedures to calculate exact unconditional $p$-values for non-inferiority/superiority and how to resolve them. Biometrical Journal 47, 37-47.

SimES, R. J. (1986). An improved Bonferroni procedure for multiple tests of significance. Biometrika 73, 751-754. 


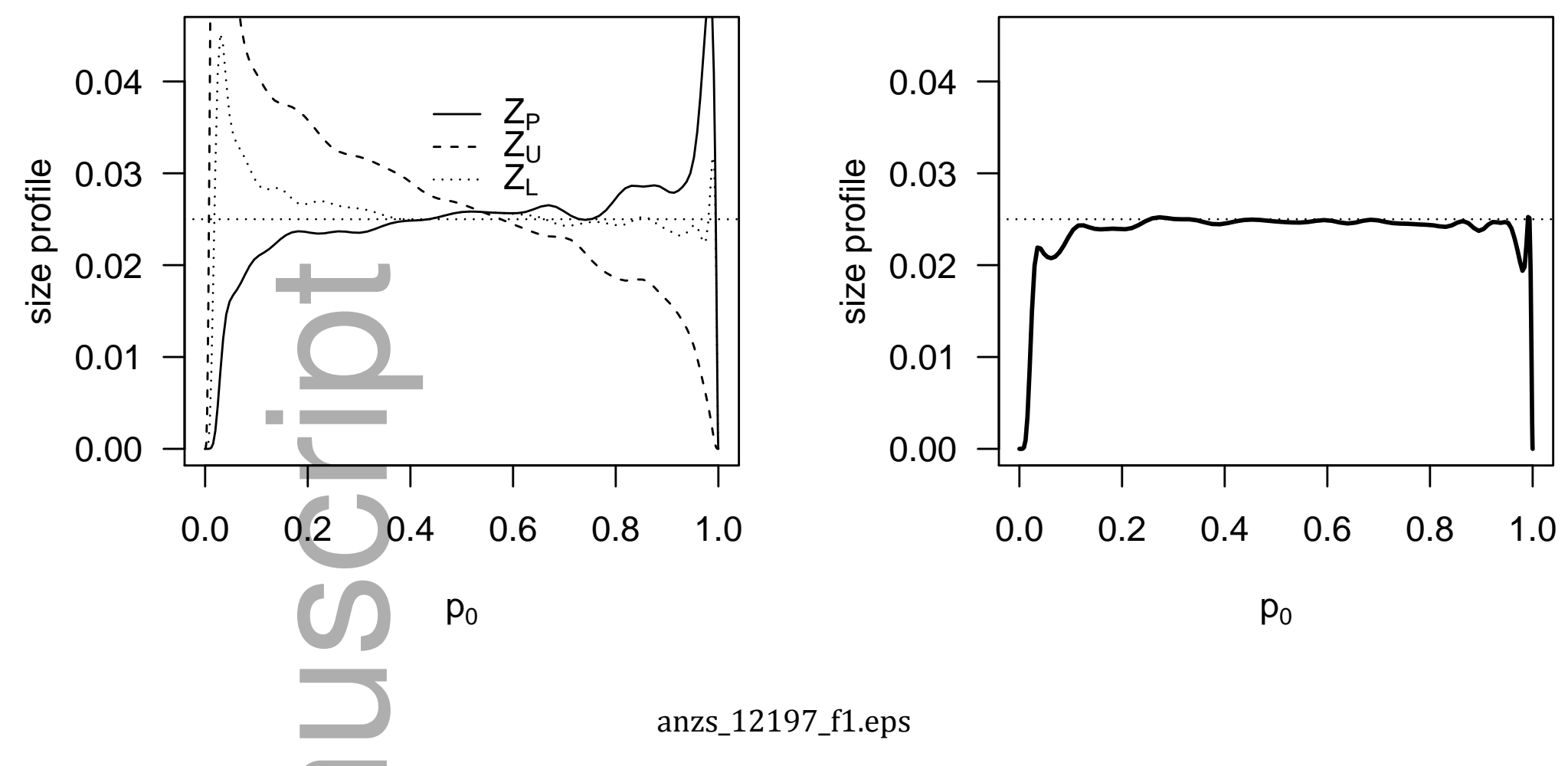

This article is protected by copyright. All rights reserved 


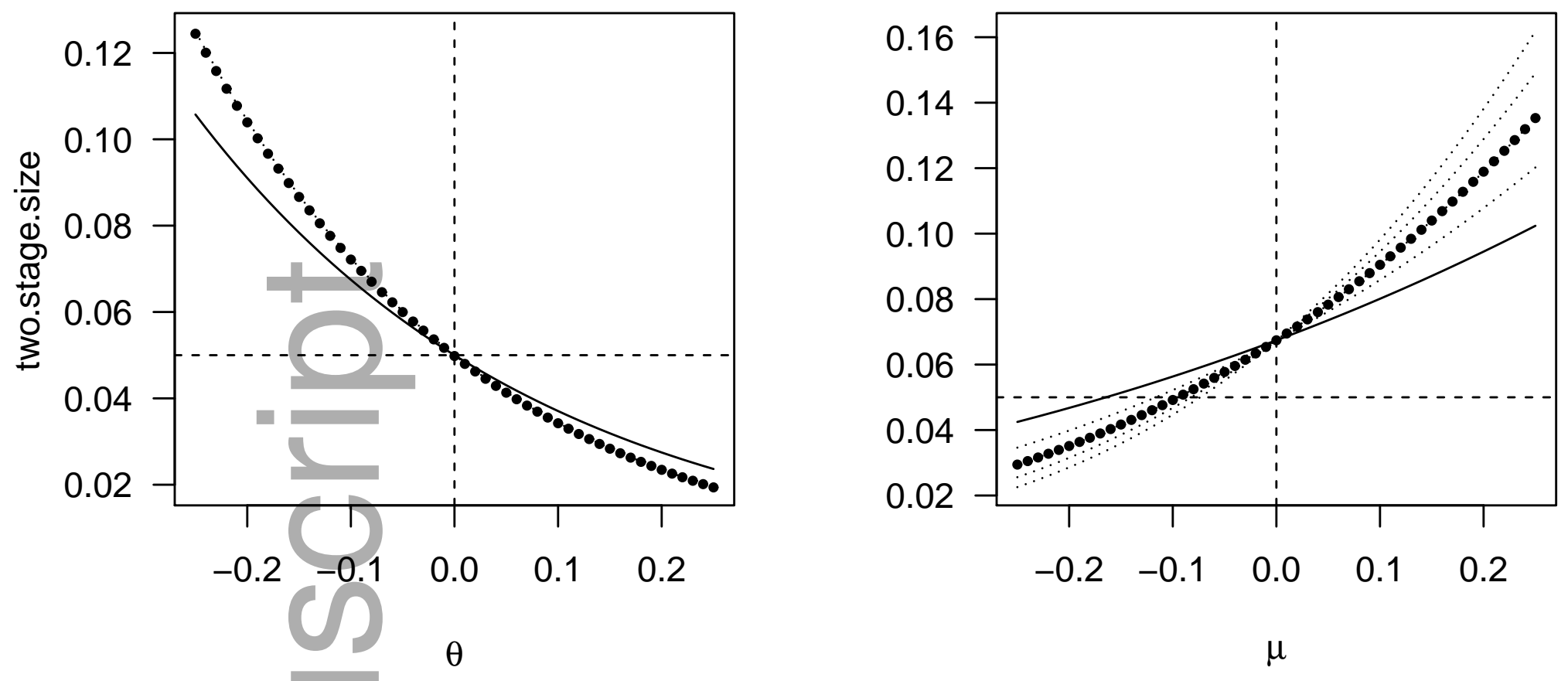

anzs_12197_f2.eps

This article is protected by copyright. All rights reserved 
nominal and actual density
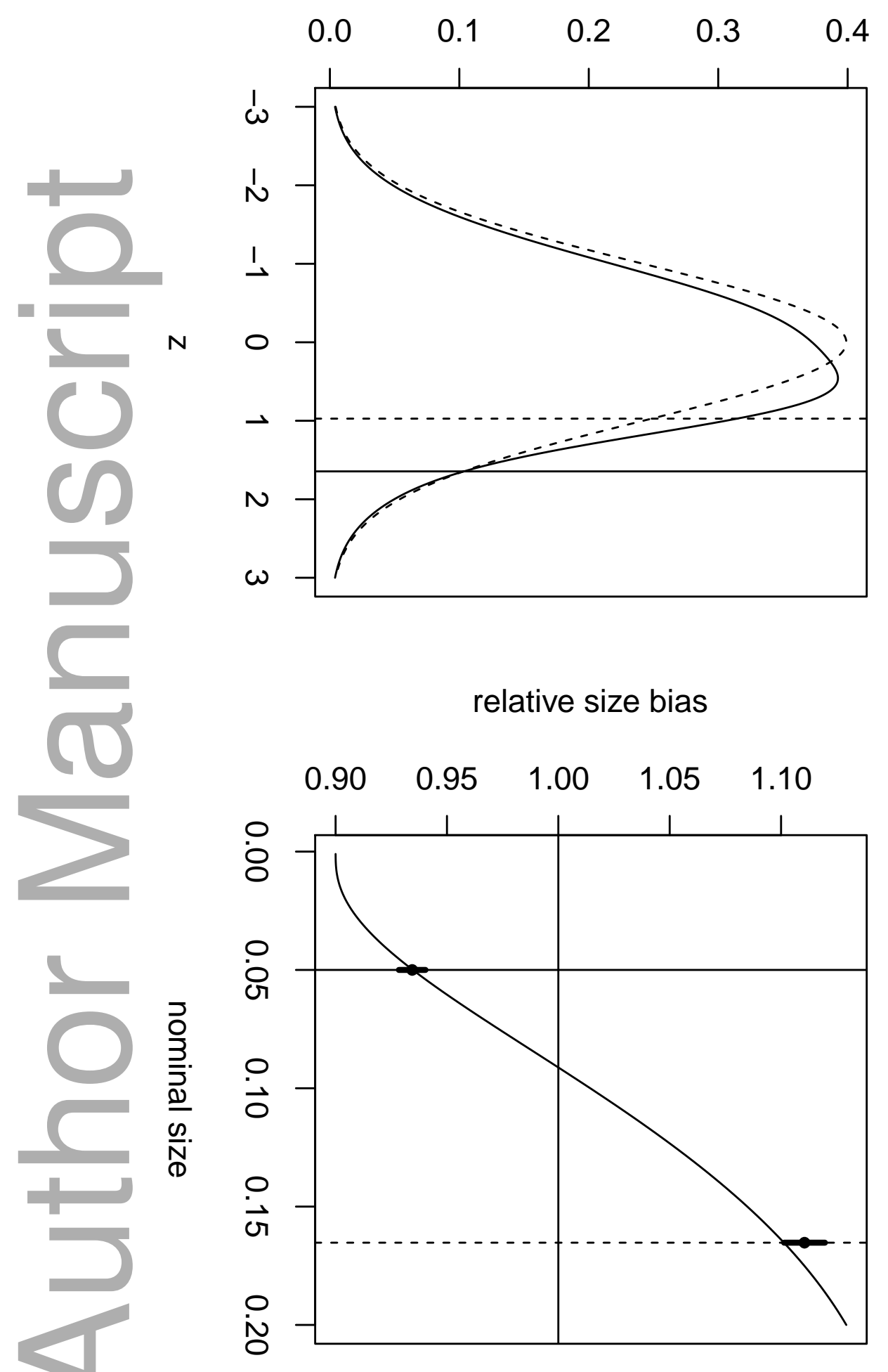

anzs_12197_f3.eps 

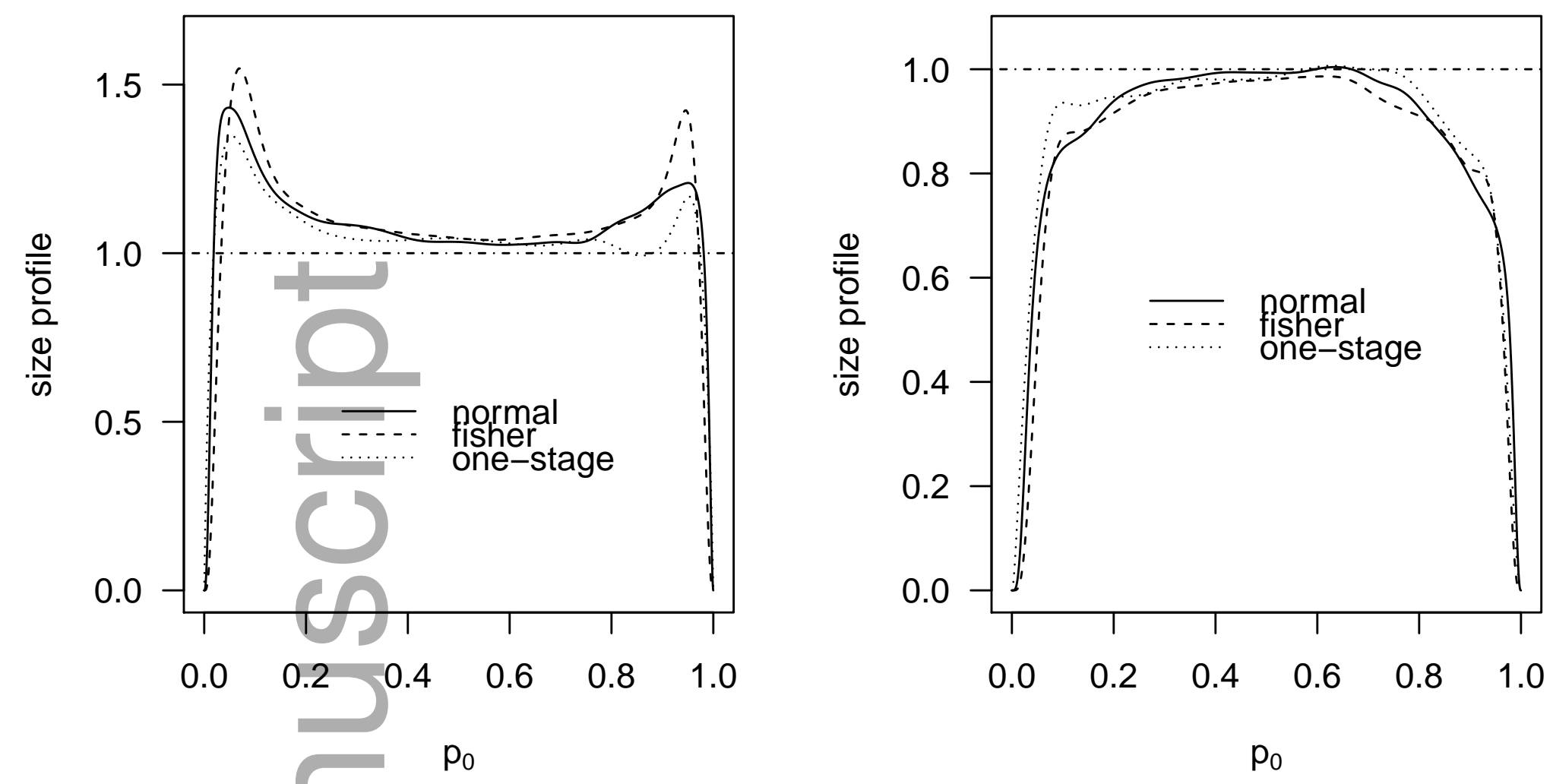

anzs_12197_f4.eps

This article is protected by copyright. All rights reserved 


\section{University Library}

\section{- M M I N E R VA A gateway to Melbourne's research publications}

Minerva Access is the Institutional Repository of The University of Melbourne

Author/s:

Lloyd, CJ

Title:

The size accuracy of combination tests

Date:

2017-09-01

Citation:

Lloyd, C. J. (2017). The size accuracy of combination tests. AUSTRALIAN \& NEW ZEALAND JOURNAL OF STATISTICS, 59 (3), pp.275-287. https://doi.org/10.1111/anzs.12197.

Persistent Link:

http://hdl.handle.net/11343/293400 